\title{
Hemangioma of Ureter: A Diagnostic Dilemma-Managed Surgically Using Robotic Platform
}

\author{
Gopal Ramdas Tak, MBBS, MS, DNB, Shashank Agrawal, MBBS, MS, \\ Mahesh Ramanlal Desai, MBBS, MS, FRCS, FRCS, Arvind Prakash Ganpule, MBBS, MS, DNB, MNAMS, \\ Abhishek Gajendra Singh, MBBS, MS, Mch, DNB, and Ravindra Bhalchandra Sabnis, MBBS, MS, Mch
}

\begin{abstract}
Ureteral hemangioma is a rare entity and few cases have been reported in the literature. Nearly all cases are diagnosed on pathologic examination postoperatively. In this study we report a 22 years young male patient presented with left-sided flank pain and hematuria. He underwent computed tomography, cystoscopy, left retrograde pyelogram, left side ureteroscopy, and urine cytology. Ureteroscopy showed a left upper ureteral narrowing with bulging mass above it. Robot-assisted segmental ureterectomy with end-to-end anastomosis over 6/26 Double-J stent was done and histopathologic examination of excised ureter reported ureteral hemangioma.
\end{abstract}

Keywords: ureter, hemangioma, robot

\section{Introduction}

$\mathbf{H}$ EMANGIOMA OF THE URINARY TRACT is a relatively unusual entity and seldom described in the literature. In the past years because of the advancement of radiologic imaging, an increase of its incidence has been recorded. Benign ureteral tumors are less frequent, but they cannot be distinguished from ones malignant before surgery. ${ }^{1}$

In this study, we describe a case of a ureteral hemangioma diagnosed by pathologic examination after robotic segmental ureterectomy. Characteristic of a ureteral hemangioma that differs from those of malignant ureteral tumors also reviewed along with its diagnosis and treatment.

\section{Case Report}

A 22 years young male patient, presented with history of left-sided flank pain, vomiting occasionally, along with three episodes of total gross hematuria, which was dark red, throughout the micturition, once associated with serpentine/ vermiform clot. His general examination was unremarkable. Baseline blood investigations were normal, including renal functions. Ultrasound examination was showing left side moderate to gross hydronephrosis till the iliac crossing; with normal opposite kidney. CT intravenous urography (CT IVU) revealed iso-dense (30-50 HU) soft tissue lesion involving the left mid-ureteral segment $(5 \mathrm{~cm}$ distal to pelvic-ureteral junction) with moderate contrast enhancement (120-130 $\mathrm{HU})$ and proximal moderate to gross hydroureteronephrosis (Fig. 1)

Retrograde pyelography showed mild narrowing seen at the L5-S1 level and another narrowing seen at the L3 level (about $1-2 \mathrm{~cm}$ ) (which was the area above and below the bulge/filling defect) (Fig. 2). Eight French ureteroscopy identified a definitive bulge seen just proximal to narrowing seen. Selective left ureteral urine cytology and wash cytology were negative. Considering the moderate enhancement in a soft tissue lesion in a left ureter we did robot-assisted segmental ureterectomy with end-to-end ureteroureterostomy along with placement of Double-J $(6 \mathrm{~F} / 26 \mathrm{~cm})$ stent. Frozen section from both proximal and distal end of the excised ureter was negative for malignancy. Pathologic examination (both gross and microscopic) (Figs. 3 and 4) revealed that it was a hemangioma of the left ureter.

\section{Discussion}

Majority of ureteral tumors are malignant and benign ureteral tumors are rare. Among benign ureteral tumor fibromyoma is most common. ${ }^{2}$ Hemangioma of the urinary tract is rare. The most common site of involvement is the kidney, followed by the urinary bladder. ${ }^{2}$ Ureteral hemangiomas being rare benign tumors and only eight cases have been reported worldwide. ${ }^{3}$ To the best of our knowledge, this

Department of Urology, Muljibhai Patel Urological Hospital, Nadiad, India. 

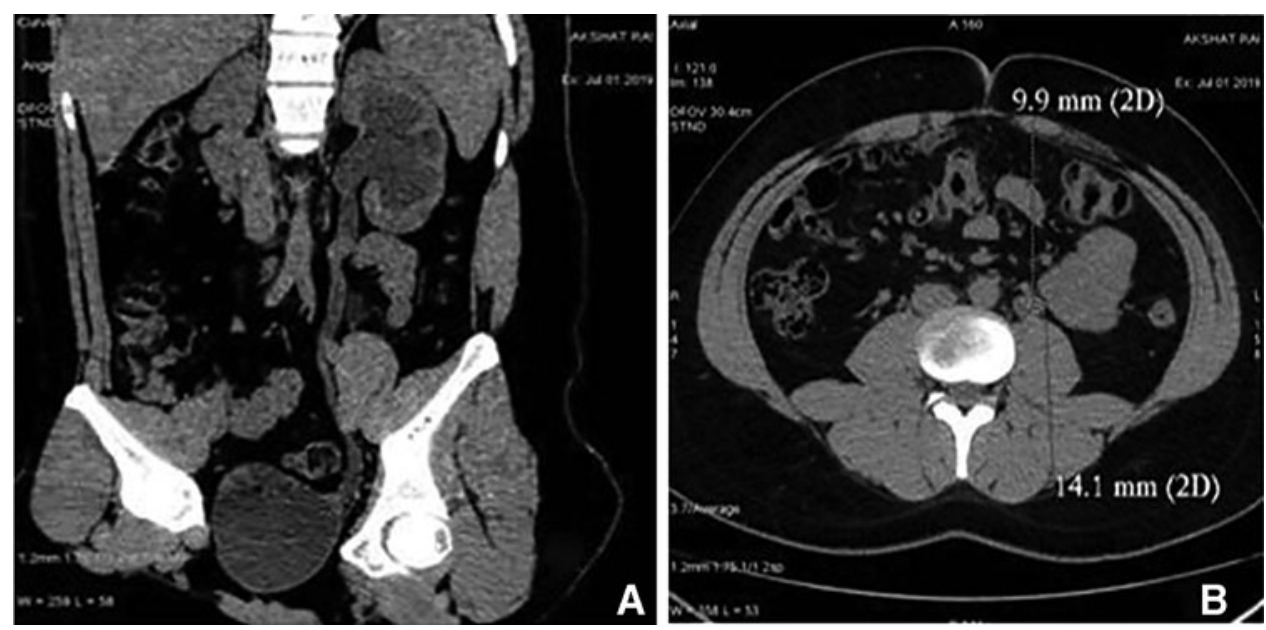

FIG. 1. (A) CT showing left hydronephrosis. (B) Mass seen in left ureter at the midureter level.

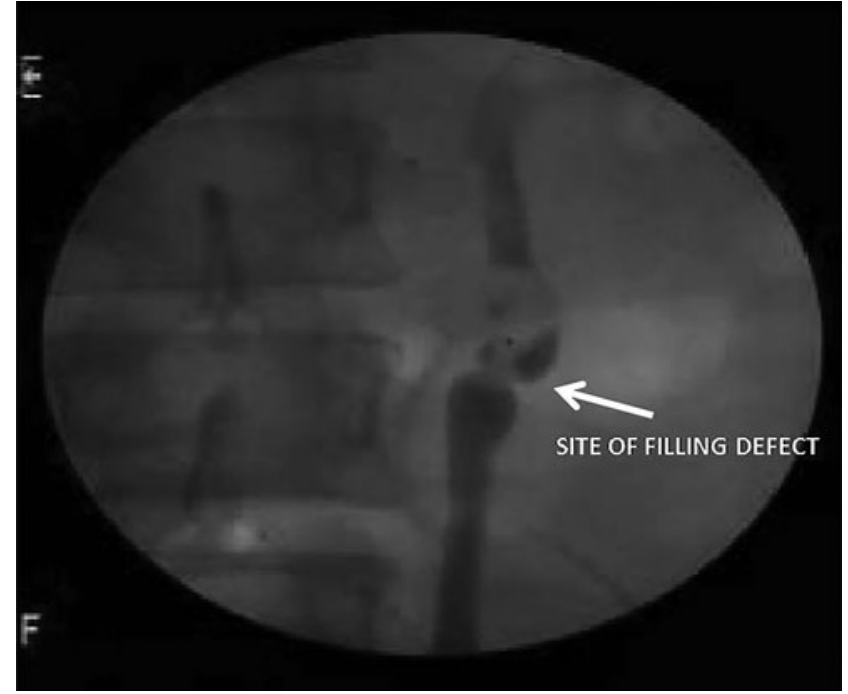

FIG. 2. Retrograde pyelogram showing left ureteral narrowing. is the first case report of ureteral hemangioma from India; that too managed surgically using robotic platform.

In 1925, Caulk described the first indisputable case of hemangioma of the urinary tract. ${ }^{4} \mathrm{He}$ described a case of telangiectatic periureteral hemangioma of the bladder wall in which the tissue formed a ring around the lower part of the ureter.

Hemangioma is a benign tumor of vascular origin, because of embryologic remains of unipotent angioblastic cells that develop in an anomalous way inside the blood vessels. Histologically, the lesion consists of lacunas, covered with endothelium, containing erythrocytes and organized thrombi.

The symptoms presented by patients are hematuria, colic type pain, and lower urinary tract symptoms. ${ }^{2}$ Hematuria occurs because of thrombus, infarction, and angiogenesis by the erosion of the urothelial lining. Flank pain may be caused by ureteral obstruction; however, some patients may be asymptomatic. In $2 \%$ cases there is a synchronous association between urethral and urinary bladder cancer. ${ }^{3}$

CT IVU is not useful diagnostic test for distinguishing ureteral benign and malignant tumor because of uncharacteristic
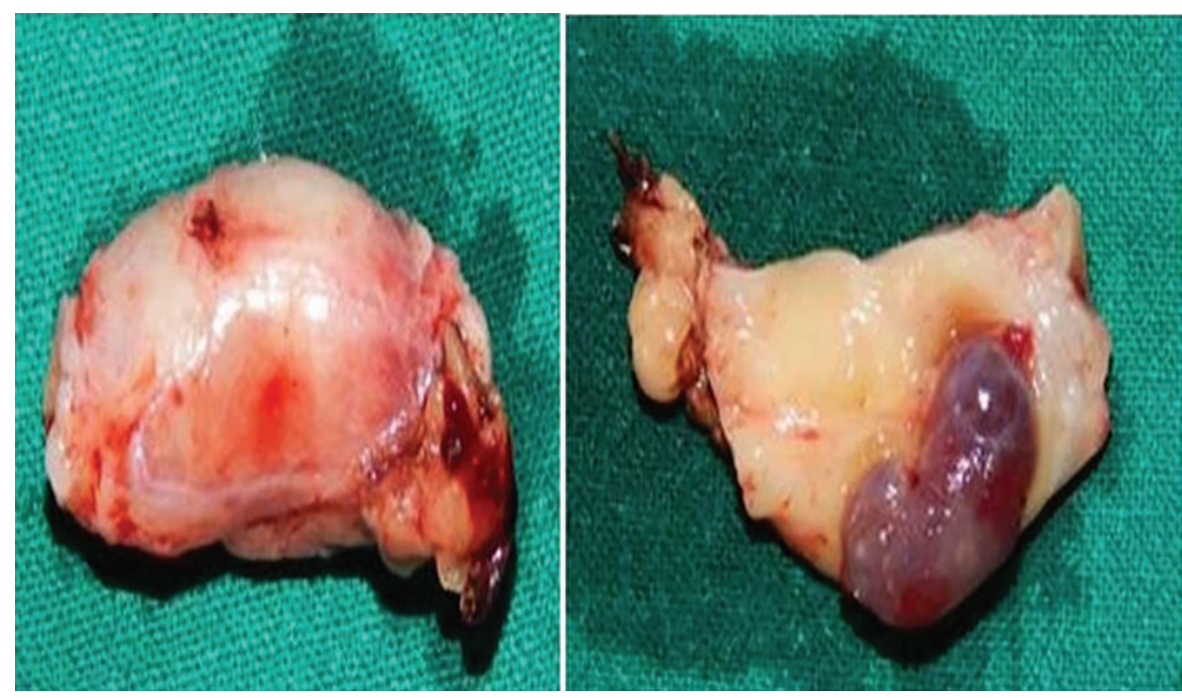

FIG. 3. Gross specimen of an excised segment of left ureter and ureter cut open showing polyploidy bulging mass. 
FIG. 4. Low- and high-power magnification showing vascular structures lined with endothelial lining cells and contains red blood cells in the lumen.

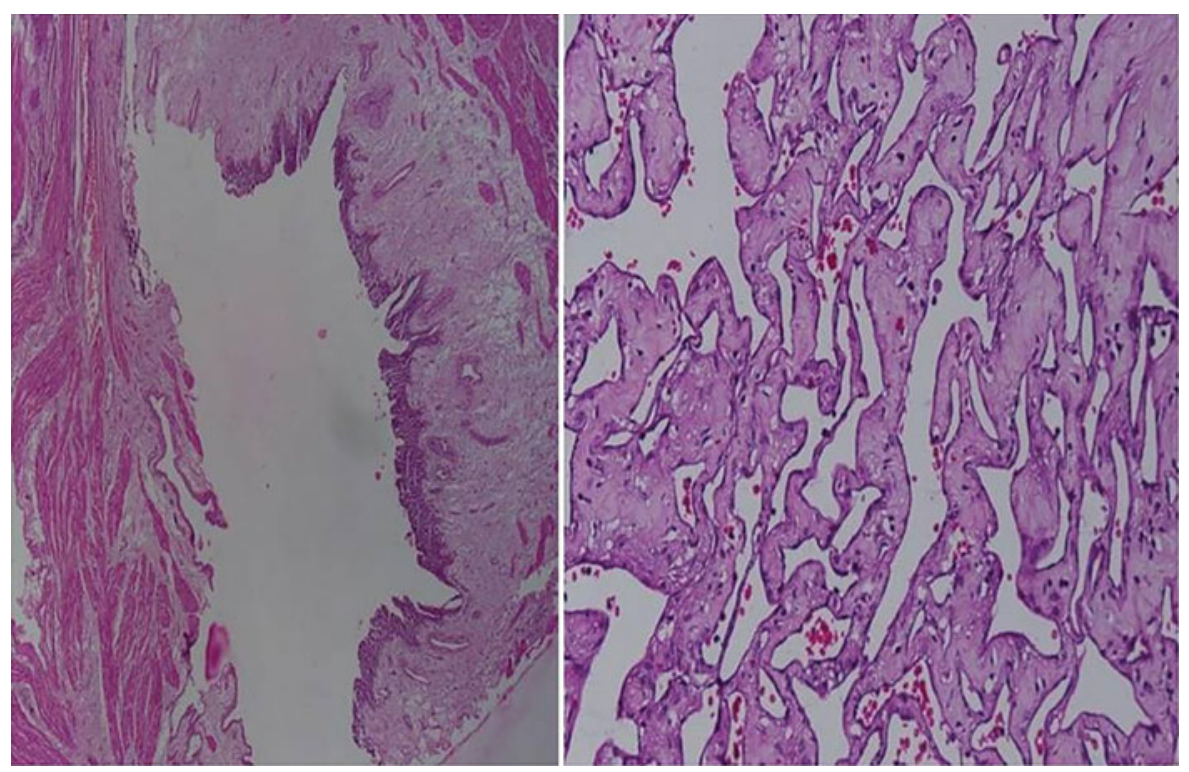

ureteral defect seen in these tests. Ureteroscopy plays a vital role in picking the ureteral hemangioma that appears as a mass with intact urothelial lining in the ureter or may protrude into the bladder as a result of ureteral peristaltic movements. Cystoscopic biopsy without significant bleeding was performed in one case of ureteral hemangioma. Majority of ureteral hemangioma are identified by pathologic examination after their removal. ${ }^{2,3}$ Role of urine cytology is crucial to rule out a malignant ureteral tumor, and the test should be performed when a ureteral tumor is suspected. In ureteral hemangioma urine cytology is normal.

In this case, patients had symptoms of left flank pain and hematuria and normal urine cytology results. Ureteroscopy revealed a bulge with intact urothelium above the narrowing located in left ureter at third lumbar vertebra. The ureteroscope could not be negotiated proximal to bulging mass, so we failed to examine the proximal ureter. CT scan showed hydronephrosis of the left kidney, as the report by Biyani et al., who described hydronephrosis with a ureteral hemangioma of the distal ureter. We considered it to be a ureteral mass and did robot-assisted left segmental ureterectomy with end-to-end ureteral anastomosis.

As per the various literatures regarding ureteral hemangiomas, the natural history of hemangioma shows spontaneous regression as a result of fibro sclerosis. Some had suggested nephrectomy and ureterectomy as a classical aggressive therapeutic approach, and others had extended the resection to the portion of bladder concerning the ureter. The chief reasons governing this method was the possibility of the development of implantation metastases, which is frequent with papillomatous tumors, and the fact that it is impossible to determine preoperatively whether the tumor is benign or malignant. Literature review has shown that nephrectomy with ureterectomy should be done for tumors of the renal pelvis and upper third of the ureter but not for tumors of the lower third of the ureter, which are in the majority and are often solitary. But few case reports had shown that the conservative operation also got equivalent outcome as the classical radical operation.
There were case reports in which patient suffering from persistent hematuria whose urography suggested for transitional-cell carcinoma of the lower ureter, which was later confirmed by a retrograde contrast study. The patient underwent nephroureterectomy and the definitive histologic examination revealed a hemangioma. In this case, the cytology was negative for malignant cells and on ureteroscopy showed bulging mass with epithelium intact and the margins of resected end of ureter were free of tumor we considered to limit ourself to segmental ureterectomy.

Till date, the cases of ureteral hemangioma were tackled with either open resection or endoscopic resection; in this study, we describe excision of ureteral hemangioma using robotic platform with added advantages of robot-assisted surgery.

\section{Conclusion}

Ureteral hemangioma is an extremely rare entity with few cases reported in the literature. Usually, it is diagnosed on pathologic examination; however, urine cytology, ureteroscopy, and intraoperative frozen margins direct the surgeon toward more conservative surgery keeping in mind the benign nature of mass. Our case report demonstrates that not all ureteral lesions are malignant and that with appropriate planning, an otherwise regretful nephroureterectomy can be avoided.

\section{Disclosure Statement}

No competing financial interests exist.

\section{Funding Information}

No funding was received for this article.

\section{References}

1. Maestroni U, Dinale F, Frattini A, Cortellini P. Ureteral hemangioma: A clinical case report. Acta Biomed 2005;76: $115-117$. 
2. Jahn H, Nissen HM. Haemangioma of the urinary tract: A review of the literature. Br J Urol 1991;68:113-117.

3. Kim SJ, Cho HJ, Ha U, Hong SH, Hwang TK, Kim JC. Cavernous hemangioma of the ureter. Korean J Urol 2009; 50:192-194.

4. Caulk JR. Haemangiomata of the bladder and ureter. Surg Gynec Obst 1925:1;49-52.

Address correspondence to: Gopal Ramdas Tak, MBBS, MS, DNB Department of Urology Muljibhai Patel Urological Hospital Nadiad 387001

India

E-mail: drgopaltak@gmail.com

\begin{tabular}{|c|}
\hline Abbreviations Used \\
$\mathrm{CT}=$ computed tomography \\
$\mathrm{HU}=$ Hounsfield units \\
$\mathrm{IVU}=$ intravenous urography
\end{tabular}

Cite this article as: Tak GR, Agrawal S, Desai MR, Ganpule AP, Singh AG, Sabnis RB (2020)

Hemangioma of ureter: a diagnostic dilemmamanaged surgically using robotic platform, Journal of Endourology Case Reports 6:3, 128-131, DOI: 10.1089/cren.2019.0148. 